Methodology We retrospectively reviewed the all cases of abdominal surgery open or laparoscopically from 20172020. Patients that was included in the Enhanced Recovery After Surgery Protocol were excluded in order to avoid bias in our outcomes. The parameters that were documented were intraoperative complications, complications during the hospitalization of the patient, readmission of patients, reoperation of patients, patients' comorbidities, the charlson comorbidity index (CCI) and body mass index(BMI). Secondary events were classified according to the Clavien Dindo classification.

Result(s)* In total 1006 patients records were reviewed. 438 $(43,3 \%)$ were in patients with advanced stage disease. Mean CCI was 3,5 and BMI 28,78. Class 1 and 2 complication rates were documented in 193 patients $(19,1 \%)$. The mean hospitalization times of these patients were 13,1 days in contrast to the uncomplicated patients that was 3,53 days.

Class 3 complications were documented in 54 cases (5,36\%). 19 cases were reoperated. The reasons for reoperation were massive hemorrhage in 3 cases, urinary tract complication in 6 cases, surgical wound dehiscence in 5 cases and 5 cases of gastrointestinal tract leakage.

Totally, 12 patients succumbed after surgery. 4 of the patients were emergency operations due to peritonitis, 2 patients succumbed due to complications from respiratory tract infections, in 3 cases from sepsis due to leakage from the gastrointestinal tract and 3 patients from cardiovascular events.

Conclusion* The increased radicality of gynecological oncology procedures increase hospitalization days as well as the perioperative morbidity and mortality.

\section{HIGH- GRADE POORLY DIFFERENTIATED SARCOMA OF UNKNOWN ORIGIN}

C Pappa*, M Abdalla, S Smith, HJ Jiang, H Soleymani Majd. , Gynaecological Oncology ، Oxford

\subsection{6/ijgc-2021-ESG0.292}

Introduction/Background* We present a rare case of highgrade poorly differentiated sarcoma. Due to the advanced nature of the disease this presented significant challenges regarding surgical management.

Methodology A 67-year-old lady with previous caecal cancer presented with significant procidentia descending $4-5 \mathrm{~cm}$ below the introitus, which was ulcerated and friable. The patient was diagnosed with Covid-19 infection prior to surgery resulting in delayed management and need for prolonged catheterisation due to tumour related urinary retention. Type 3 radical hysterectomy was performed after we devascularized the uterus by division of the uterine artery at origin- the uterus was completely inverted pulling the round ligament, ureters and iliac vessels. Given the fact that it was not clarified whether it is cervical or uterine malignancy and following the LACC trial, we completed all steps via laparoscopy and colpotomy was performed via a $10 \mathrm{~cm}$ transverse suprabupic incision to avoid spillage.

Result(s)* Histopathology indicated high-grade poorly differentiated sarcoma of unknown origin extending from beyond the cervix within the vaginal vault inwards, enveloping the cervical and lower uterine walls. The endometrium was inactive, the myometrium did not contain any leiomyomatous or stromal lesion and both adnexa were normal. Catheter was removed 4 months after her presentation.

Conclusion* The cornerstone of the optimal management of such rare and complicated cases is a combination of continuous multidisciplinary team consensus, precise surgical management and close surveillance of the patient.

\section{LAPAROSCOPIC REPAIR OF LEFT}

${ }^{1} \mathrm{C}$ Yalcinkaya*, ${ }^{2} \mathrm{~S}$ Bostan. 'Umraniye Research Hospital, Gynaecological Oncology, Istanbul, TURKEY; ${ }^{2}$ Umraniye Research Hospital, ObstetricsandGynaecology, Istanbul, TURKEY

\subsection{6/ijgc-2021-ESGO.293}

Introduction/Background* Renal vein injury is a rare but dangerous complication of paraaortic lymphadenectomy, and if happens during laparoscopic procedure; oftenly requires conversion to urgent laparotomy. In this video case, we report a circumaortic left renal vein injury and its laparoscopically repair.

Methodology In our case, there was a circumaortic left renal vein. A normal left renal vein passing anterior to the aorta and an accessory left renal vein passing posterior to the aorta. This retroaortic arm injured during paraaortic lymphadenectomy. The injured area was repaired laparoscopically with buldog clamp and polypropylene sutures. The patient was 63 year old female with endometrial carcinosarcoma.

Result(s)* Our procedure was completed succesfully after the repair as planned. There was no bleeding after repair. The patient was discharged postoperative day 3 .

Conclusion* Major vessel injuries during laparoscopic paraaortic lymphadenectomy are a common cause of urgent laparotomy. In this report, we managed the injury laparoscopically.

\section{OVARIAN CANCER IN BRCA 1 AND BRCA 2 MUTATIONS CARRIERS, CLINICOPATHOLOGYCAL FEATURES}

${ }^{1} \mathrm{~A}$ Laura, ${ }^{2} \mathrm{MDLR}$ Oliver*, ${ }^{2} \mathrm{E}$ Felipe Pardo, ${ }^{2} \mathrm{~J}$ Montero Olmeda, ${ }^{1} \mathrm{~A}$ Sofia, ${ }^{3} \mathrm{M}$ De Miguel Reyes, ${ }^{2} \mathrm{C}$ Alvarez, ${ }^{2} \mathrm{~A}$ Tejerizo. ${ }^{1}$ Hospital Universitario 12 de Octubre, Gynecologycal and Obstetrics department, Breast unit; ${ }^{2}$ University Hospital October 12, Gynecologycal oncology, Madrid, Spain; ${ }^{3}$ Hospital Universitario 12 de Octubre, Genetics department

\subsection{6/ijgc-2021-ESGO.294}

Introduction/Background* Mutations in the BRCA1 or BRCA2 susceptibility genes are associated with most hereditary breast cancers with an identified pathogenic variant. These genes are implicated in about $5-10 \%$ of women with familial ovarian cancer. The aim of this study is to evaluate clinicopathological features of ovarian cancer diagnosed in BRCA1/2 mutations carriers.

Methodology A retrospective descriptive study of all women diagnosed with BRCA gene mutations at a high-volume center between January 2007 and October 2020 was performed.

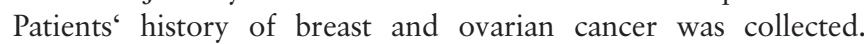
IBM SPSS Statistics ${ }^{\circledR}$ v25.0 was used for statistical analyses.

Result(s)* We included 165 patients diagnosed with BRCA1 and BRCA2 mutation, 114 prophylactic salpingo- 
oophorectomies were performed. There was 21 diagnosis of ovarian cancer ( 7 patients among BRCA1 carriers and 14 patients among BRCA2 carriers). The mean age at diagnosis was 48.9 (SD 18.1) and 54.4 (SD 10.)9 years for BRCA1 and BRCA2 respectively $(\mathrm{p}=0.475)$.

The most frequent histology was high-grade serous ovarian adenocarcinoma (19 cases), 1 endometrioid carcinoma and 1 serous tubal adenocarcinoma. At the time of diagnosis, the most frequent stage was IIIC (9 cases) followed by stage IV (5 cases).

Regarding the treatment received, 11 primary surgeries were performed, in which primary complete cytoreduction was obtained in 10 of the cases. The other 10 cases received neoadjuvant chemotherapy (NACT) and surgery was subsequently performed. The median follow-up was 89 (EQR 34.5183.5) months. During this follow-up 6 patients died

Conclusion* To be a BRCA mutation carrier is associated with an increased risk of ovarian cancer at earlier ages than the general population, with no significant differences between the two types of mutation. The most frequent histology was highgrade serous ovarian adenocarcinoma and the most frequent stage at diagnosis was IIIC.

\section{BRCA-MUTATION-ASSOCIATED FALLOPIAN TUBE CARCINOMA}

${ }^{1} V$ Benito*, ${ }^{2} \mathrm{C}$ Molo, ${ }^{3} \mathrm{P}$ Garcia, ${ }^{4} \mathrm{~A}$ Lubrano. ${ }^{1}$ Vithas Las Palmas, Gynecology Oncology, Las Palmas de Gran Canaria, Spain; ${ }^{2}$ Vithas Las Palmas, Gynecology and Obstetrics, Las Palmas de Gran Canaria, Spain; ${ }^{3}$ Eurofins Megalab Canarias, Pathology, Las Palmas de Gran Canaria, Spain; ${ }^{4}$ C.H.U. Insular-Materno Infantil, Gynecology and Obstetrics, Las Palmas de Gran Canaria, Spain

\subsection{6/ijgc-2021-ESG0.295}

Introduction/Background* Fallopian tube carcinoma is a very rare tumor, comprising less than $1 \%$ of all gynecologic cancers and found primarily in postmenopausal women. Women with fallopian tube carcinoma are reported to have a higher frequency of inherited BRCA mutations than those with ovarian carcinoma or primary peritoneal carcinoma, approximately $30 \%$ of women with fallopian tube cancer have a mutation in BRCA1 or BRCA2. Described risk factors for BRCA mutations in these patients, are younger age (under 60) and women with a family history of breast or ovarian cancer.

Methodology The patients' clinical, pathological, and imaging data are presented. Likewise, the most relevant surgical videos are also presented.

Result(s)* We describe the case of a 58-year-old nulliparous patient with a family history of ovarian cancer who underwent surgery for suspected ovarian cancer with carcinomatosis. Complete debulking surgery was achieved after pelvic peritonectomy, total hysterectomy, bilateral salpingo-oophorectomy, radical omentectomy, right diaphragmatic peritonectomy and exeresis of several paraaortic enlarged lymph nodes. Histological examination showed the presence of a high-grade serous fallopian tube cancer, FIGO stage IIIB, TNM pT3b N0 M0. Mutational analysis confirmed the presence of a deleterious mutation in BRCA-1. She underwent platinum-based chemotherapy and maintanence therapy with olaparib. Currently, 3 years after treatment, she is alive without evidence of disease.

Conclusion* Given the high frequency of BRCA mutation, all patients diagnosed with invasive fallopian tube cancer should be considered candidates for genetic testing. Likewise, patients with germline BRCA1 gene mutations in whom a prophylactic oophorectomy is performed, removal of fallopian tubes should be strongly considered.

\section{STEP BY STEP TOTAL OMENTECTOMY AND ANATOMICAL BOUNDARIES ON LIVE PATIENT (OPEN TECHNIQUE)}

I Selcuk*, HR Yalcin. Ankara Şehir Hastanesi, Turkey

\subsection{6/ijgc-2021-ESG0.296}

Introduction/Background* To demonstrate the proper anatomical boundaries and surgical technique for total omentectomy on a live patient.

Methodology This surgical video was recorded at Ankara City Hospital, an ESGO Accredited Gynecologic Oncology Center. Result(s)*

- The greater omentum attaches the stomach to the transverse colon. The double fold of the peritoneum encircling the stomach turns over itself and attaches to the transverse colon and transverse mesocolon. By the way, the greater omentum has four peritoneal folds.

- The greater omentum lies cranially from the greater curvature of the stomach and proximal duodenum, traverses over the transverse colon between the hepatic flexure at the right side and splenic flexure at the left side, and afterwards lies over the small intestines covering the whole abdominal cavity.

- Firstly, the posterior peritoneal fold covering the omentum which attaches to the transverse colon is cut, so an avascular plane is identified superior to the transverse mesocolon.

- Secondly, after cutting the posterior peritoneal fold of the omentum between the hepatic flexure and splenic flexure (the attachments at the level of hepatic and splenic flexure are dissected), the transverse colon is grasped caudally, and the omentum is grasped cranially. By the way, the gastrocolic space is easily dissected and developed. As a consequence, the omentum is dissected from the transverse mesocolon.

- The stomach and the transverse colon are separated, the omentum is found attached to the stomach, and the bursa omentalis is accessed. Afterwards, the omentum is excised from the hepatic flexure to the splenic flexure below the level of the greater curvature. If there is not any gross tumor nodule at this part, the gastro-epiploic vessels are preserved.

- While excising the omental structure between the stomach and spleen, care should be taken for short gastric vessels lying here, especially at the cranial part of this area. Besides, close to the splenic hilum, the omental tissue should be discriminated from the tail of the pancreas.

Conclusion* Developing the gastrocolic space is crucial while performing a total omentectomy. In addition, the peritoneal folds of the omentum should be dissected clearly and gently. 